\title{
An EnERgy Aware Geocast Algorithm using 2-HOP NEIGHBOUR KNOWLEDGE IN SENSOR NETWORKS
}

\author{
Young-Chul Shim ${ }^{1}$ and Sehun Noh $^{2}$ \\ ${ }^{1}$ Department of Computer Engineering, Hongik University, Seoul, Korea \\ shimecs.hongik.ac.kr \\ ${ }^{2}$ GuardianNet, Seoul, Korea \\ sehun1016@hanmail.net
}

\begin{abstract}
Geocast is a mechanism which sends a packet to all the sensor nodes in a certain geographic area. It enables one to collect information from the designated area within a sensor network. Because sensor nodes are operating with limited power source and sending/receiving packets are most energy consuming operations for sensor networks, geocast algorithms should be energy efficient. In this paper we introduce a new geocast protocol which minimizes energy usage and overhead by utilizing the knowledge on the neighbours and their energy levels. We evaluate the performance of the proposed protocol with simulation.
\end{abstract}

\section{KEYWORDS}

Sensor Networks, Geocast, Energy Efficiency, Broadcast, Performance Evaluation

\section{INTRODUCTION}

Sensor networks have been used for a wide range of applications including environment monitoring, traffic surveillance, military sensing and information gathering. Their main purposes are to monitor an area, including detecting, identifying, localizing, and tracking one or more objects of interest. A sensor network consists of one or multiple data centers called a sink node and many low-cost and low-powered sensor devices, called sensor nodes. Each sensor node has the ability of sensing data, processing data, and communicating with others via radio transceivers. The sink node, equipped with a database system, sends geocast packets, which are queries or control commands, to sensor nodes and collects information from sensors. The communication between the sink and sensor nodes relies on the relay by intermediate sensor nodes [1].

Because sensor nodes are microelectronic devices, they can only be equipped with a limited power source. Therefore, energy conservation becomes the most important issue when developing routing protocols for sensor networks. Techniques such as in-network data aggregation are needed to reduce energy consumption in sensor nodes.

In sensor networks a group of sensor nodes in a certain geographic region may cooperate to monitor an object within that region. So broadcast to all the sensor nodes in that region becomes essential mechanism. Geocast, a variant of conventional broadcast, sends packets to all the nodes within a specified geographical region. To determine the geocast group membership, each node is 
required to know its own physical location i.e., its geographic coordinates, which may be obtained using a system such as the GPS(Global Positioning System)[2].

In general geocast routing consists of two phases as in Figure 1. In the first phase a command packet is delivered from the sink node to one or more nodes in the geocast region. Then the packet is broadcast to all the nodes in the geocast region. Although, most proposed geocast protocols focus on the protocol for the first phase and assume the use of flooding for the second phase. GEAR(Geographic and Energy Aware Routing) algorithm is one notable geocast protocol for sensor networks[3]. It uses energy aware neighbour selection to route packet towards the geocast region and uses Recursive Geographic Forwarding algorithm to disseminate the packet inside the geocast region. During the first phase, when a node receives a command packet, among its neighbours GEAR picks the next hop minimizing the cost which is the combination of the distance to the geocast region and the consumed energy. During the second phase, GEAR disseminates the packet to geocast nodes using a recursive routing algorithm. GEAR proposes an energy efficient protocol for the first phase, but the applicability of its second phase protocol is limited because it can be applied only to rectangular-shaped areas.

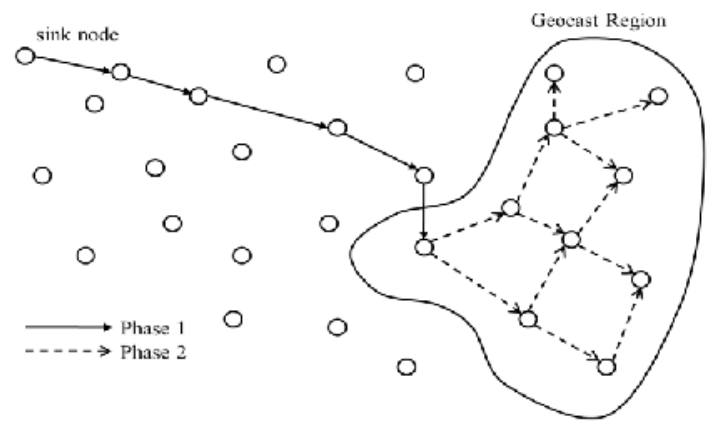

Figure 1. 2 phases in geocast routing

In this paper we propose a new energy efficient geocast protocol. We assume the use of algorithms like GEAR for the first phase of the geocast protocol and focus only on the second phase. We propose an energy efficient protocol for disseminating a geocast packet in the geocast region. We assume that sensor nodes are not mobile and have knowledge of not only 1-hop neighbours but also 2-hop neighbours. We also assume that each sensor node knows the energy level of its 1-hop neighbours. We use the knowledge of 1-hop neighbours, 2-hop neighbours, and the energy levels of 1-hop neighbours to design an energy efficient geocast algorithm. We evaluate the performance of the proposed protocol through simulation along with 2 other protocols.

The rest of the paper is organized as follows. The next section surveys related works. Section 3 describes the proposed protocol. Section 4 provides the performance evaluation results and is followed by the conclusion in Section 5 .

\section{RELATED WORKS}

Yao et al classified geocast protocols into three categories: flooding-based protocols, routingbased protocols, and cluster-based protocols[2]. But the protocols compared by them focus only on the first phase, simply use flooding mechanism for the second phase, or assume specific shapes for the geocast region. Therefore, their results have little applicability for our purposes. 
There have been many research works on network wide broadcasting in MANETs and these works can be applied to broadcasting in a geocast region in sensor networks. We briefly survey broadcasting techniques in MANETs.

Broadcasting techniques in MANETs are classified into four categories: simple flooding, probability based methods, area based methods, and neighbour knowledge methods[4].

The algorithm for Simple Flooding starts with a sink node broadcasting a packet to all neighbours. Each of those neighbours in turn rebroadcasts the packet exactly once and this continues until all reachable network nodes have received the packet[4].

Probability based methods use some basic understanding of the network topology to assign a probability to a node to rebroadcast. There are a probabilistic scheme and a counter-based scheme in this category[5]. The probabilistic scheme is similar to flooding, except that nodes only rebroadcast with a predetermined probability. In the counter-based scheme, upon reception of a previously unseen packet, the node initiates a counter with a value of one and sets a RAD(which is randomly chosen between 1 and Tmax seconds). During the RAD, the counter is incremented by one for each redundant packet received. If the counter is less than a threshold value when the RAD expires, the packet is rebroadcast. Otherwise, it is simply dropped[4].

Area based methods assume nodes have common transmission distances: a node will rebroadcast only if the rebroadcast will reach sufficient additional coverage area. There are the distance-based scheme and the location-based scheme in this category[5]. In the distance-based scheme, a node compares the distance between itself and each neighbour node that has previously rebroadcast a given packet. Upon reception of a previously unseen packet, a RAD is initiated and redundant packets are cached. When the RAD expires, all source node locations are examined to see if any node is closer than a threshold distance value. If true, the node doesn't rebroadcast. In the location-based scheme, each node must have means to determine its own location, e.g., a GPS. Whenever a node originates or rebroadcasts a packet, it adds its own location to the header of the packet. When a node initially receives a packet, it notes the location of the sender and calculates the additional coverage area obtainable were it to rebroadcast. If the additional area is less than a threshold value, the node will not rebroadcast, and all future receptions of the same packet will be ignored. Otherwise, the node assigns a RAD before delivery. If the node receives a redundant packet during the RAD, it recalculates the additional coverage area and compares that value to the threshold[4].

Neighbour knowledge methods maintain state on their neighbourhood, which is used in the decision to rebroadcast. There are Flooding with Self Pruning[6], Scalable Broadcast Algorithm(SBA)[7], Dominant Pruning[6], Multipoint Relaying[8], Ad Hoc Broadcast Protocol(AHBP)[9], Connected Dominating Set-Based Broadcast algorithm[10], and Lightweight and Efficient Network-Wide Broadcast(LENWB)[11] in this category. Among them, we describe the first two protocols. The Flooding with Self Pruning protocol requires that each node has knowledge of its 1-hop neighbours, which is obtained via periodic "Hello" packets. A node includes its list o known neighbours in the header of each broadcast packet. A node receiving a broadcast packet compares its neighbour list to the sender's neighbour list. If the receiving node would not reach any additional nodes, it refrains from rebroadcasting: otherwise the node rebroadcasts the packet. SBA requires that all nodes have knowledge of their neighbours within a two hop radius. This neighbour knowledge coupled with the identity of the node from which a packet is received allows a receiving node to determine if it would reach additional nodes by rebroadcasting. 2-hop neighbour knowledge is achievable via periodic "Hello" packets: each "Hello" packet contains the node's identifier and the list of known neighbours. After a node 
receives a "Hello" packet from all its neighbours, it has 2-hop topology information centered at itself.

\section{Description of the Proposed Protocol}

As we explained in the introduction, we assume that geocast protocol consists of two phases. The initial geocast packet issued from the sink node is routed to a certain sensor node in the geocast region using protocols as in GEAR and then the packet is distributed to all the sensor nodes in the geocast region. We focus only on the second phase of the protocol in this paper.

As a sensor node consumes energy the most when it transmits and receives a packet, we try to minimize the number of sensor nodes which forward the geocast command in the geocast region. We also consider the energy level of sensor nodes when we select the sensor nodes which will forward the geocast command. A sensor node is allowed to forward a command only if it has enough energy left in it. We aim to achieve these goals by making sensor nodes utilize knowledge of their 1-hop and 2-hop neighbours and energy levels of its 1-hop neighbours while distributing geocast packets in the geocast region. In this paper we assume that after a sensor network is deployed, a new sensor node cannot be added but a sensor node can fail to function probably due to energy exhaustion and, therefore, leave the sensor network. When a sensor network is deployed initially, all the sensor nodes broadcast a hello message to its 1-hop neighbours. The initial hello message also includes the geographic location of the sending node and its energy level. After exchanging the initial hello messages, every node knows the identity and location of its 1-hop neighbours. Then every node broadcasts to its 1-hop neighbours the second hello message which includes the identities and locations of the sending node's 1-hop neighbours. After exchanging the second hello messages, every node knows the identity and location of 2-hop neighbours. Then sensor nodes go into the normal operating mode, during which every node sends a hello message to its 1-hop neighbours periodically to inform its liveness, its energy level, and identities of failed neighbours, if any.

Now we explain the outline of the protocol which broadcasts a geocast packet in a geocast region. A geocast node $\mathrm{N}$ receives from another node $\mathrm{M}$ a geocast packet consisting of a query or command, a geocast region description, and a forwarder set. The query/command and the geocast region description have been originally issued from the sink. When a geocast node broadcasts the packet of its 1-hop neighbours, it adds its forwarder set to the packet. Among its 1-hop neighbours, a node selects some nodes that are invited to rebroadcast the packet and the set of these selected odes is called the forwarder set. If the packet is new to $\mathrm{N}$ and $\mathrm{N}$ is in M's forwarder set, $\mathrm{N}$ calculates its own forwarder set, includes it in its geocast packet, and broadcasts the packet. This process is continued until no geocast node can find a non-empty forwarder set.

The process of calculating a forwarder set may consist of two steps. In the first step, the range confinement step, the range in which a node's 1-hop neighbours and 2-hop neighbours will be considered is confined and in the second step, the node selection step, the forwarder set is calculated within the confined range.

In Figure 2, we assume that sensor node $\mathrm{N}$ received a geocast packet from the node M. To calculate N's forwarder set, we have to consider N's 1-hop neighbours and 2-hop neighbours. But as in Figure 2 (a), we can basically eliminate M's 1-hop neighbour range from consideration, because all the nodes in M's 1-hop neighbour range must have received the geocast packet. We call this strategy the basic confinement. In Figure 2 (b) we draw a line from $\mathrm{M}$ to $\mathrm{N}$ and then draw a vertical line at $\mathrm{N}$. We assume that with a high probability nodes which are located to the left of this vertical line have received the geocast packet already and, therefore, take these nodes out of consideration. We call this strategy the advanced confinement. So when we calculate N's 
forwarder set in the next step, we consider 1-hop neighbours in the gray area and 2-hop neighbours in the hatched area.

For the second step, two greedy methods have been proposed in the past $[6,12]$. In Shim's method, a sensor node N's 1-hop geocast neighbours is checked for the inclusion in N's forwarder set from the farthest node (from N) to the closet node (from N)[12]. In Lim's method, a sensor node N's 1-hop geocast neighbours is checked from the node which has the most number of 1-hop neighbours to the node which has the least number of 1-hop neighbours[6]. We call Shim's method to be the longest distance first method and Lim's method to be the most neighbours first method. Following is the pseudo-code description of these two methods.

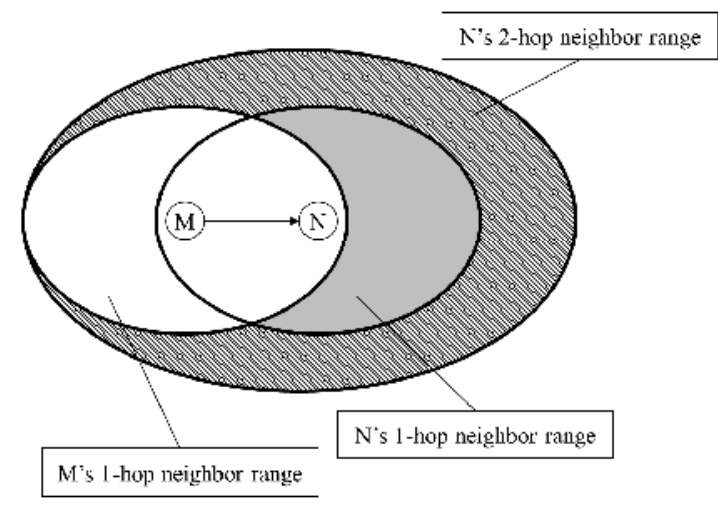

(a) Basic confinement

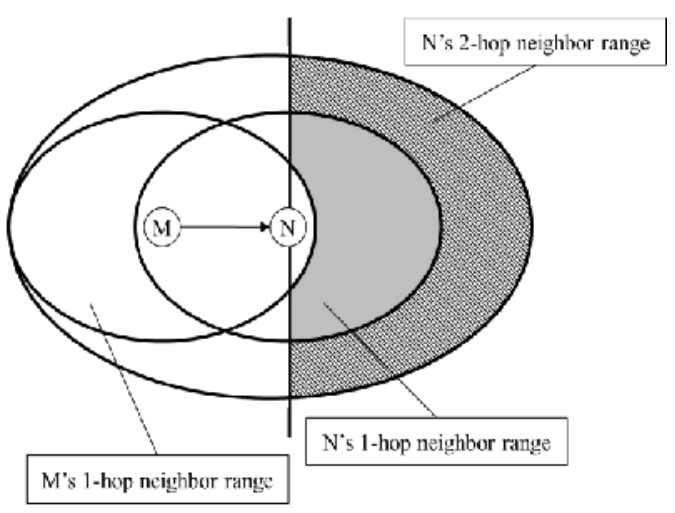

(b) Advanced confinement

Figure 2. Range confinement strategies

Set FWRD to be an empty set; /* FWRD is N's forwarder set */

Set $2 \mathrm{H}-\mathrm{GN}$ to be an empty set; /*2H-GN is N's 2-hop geocast neighbour set */

Sort N's 1-hop neighbours and sort them in $1 \mathrm{H}-\mathrm{GN}$;

/* In Shim's method, neighbours are sorted in descending order */

$1 *$ of their distance from $\mathrm{N} * /$

/* In Lim's method, neighbours are sorted in descending order */

$/ *$ of the number of their 1-hop neighbours */

While (1H-GN is not empty) \{

Remove the first node $\mathrm{P}$ in $1 \mathrm{H}-\mathrm{GN}$;

If ((P's 1-hop geocast neighbour $\cap 2 \mathrm{H}-\mathrm{GN})$ is not empty) \{

$$
\begin{aligned}
& 2 \mathrm{H}-\mathrm{GN}=2 \mathrm{H}-\mathrm{GN} \cup \mathrm{P} \text { 's 1-hop geocast neighbour; } \\
& \text { Add } \mathrm{P} \text { to FWRD }\} \text { \} }
\end{aligned}
$$

We explained two strategies for the range confinement: basic confinement(BC) and advanced confinement(AC). The we described two selection methods: longest distant first(LDF) and most neighbour first(MNF). Then we can combine these strategies and have 4 algorithms for calculating the forwarder set as follow:

- BC-LDF: basic confinement and longest distance first

- AC-LDF: advanced confinement and longest distance first 
- BC-MNF: basic confinement and most neighbour first

- AC-MNF: advanced confinement and most neighbour first

In [13], it is shown that AC-MNF has the best performance among 4 methods, and we consider only AC-MNF in this paper.

But AC-MNF does not consider the energy level of neighbour nodes and now we explain how we modify AC-MNF and consider the energy level when selecting the forwarding set. To consider the energy level of sensor nodes, we introduce the concept of the remaining energy ratio as follows. If a sensor node $\mathrm{N}$ had an initial energy level $\mathrm{E}_{\mathrm{ini}}(\mathrm{N})$ and the current energy level $\mathrm{E}_{\text {cur }}(\mathrm{N})$, the node $\mathrm{N}$ 's remaining energy ratio $\mathrm{E}_{\mathrm{rer}}(\mathrm{N})$ is defined as follows.

$$
\mathrm{E}_{\text {rer }}(\mathrm{N})=\mathrm{E}_{\text {cur }}(\mathrm{N}) / \mathrm{E}_{\text {ini }}(\mathrm{N})
$$

Erer(N) starts with the value 1 and converges to 0 as the node N's energy is exhausted. Then the third step of the above algorithm, which sorts 1 hop neighbours, is modified. For each neighbour node $\mathrm{N}$, the value of (the number of the node N's 1-hop neighbours) * $\left(\mathrm{E}_{\mathrm{rer}}(\mathrm{N})\right)$ is calculated and the neighbour nodes are sorted in the descending order of this value. With this modification, a neighbour node can be included in a forwarder set only if it has both many 1-hop neighbours and enough remaining energy. We call this modified algorithm to be the EA(Energy-Aware)-ACMNF algorithm.

\section{Performance Evaluation}

To evaluate the performance of the proposed algorithm EA-AC-MNF, we compare its performance with the flooding algorithm and the AC-MNF algorithm through simulation.

\subsection{Experimentation Environment}

We assume that sensor nodes are located on an area of a $2000 \mathrm{~m} * 2000 \mathrm{~m}$ square. 400 sensor nodes are randomly located over this area. The geocast region has the shape of a $500 \mathrm{~m} * 500 \mathrm{~m}$ square and is located at the center of the sensor network area. We assume that the communication range of a sensor node is $15 \mathrm{~m}$, its sensing range is $8 \mathrm{~m}$, and each packet is 100 byte long. After a geocast command is delivered, each sensor node in the geocast region periodically senses data and reports it. Reported data is delivered in the reverse direction of the geocast command delivery. The simulator is programmed with Visual $\mathrm{C}++6.0$ over Windows XP.

We use the following 3 metrics to compare performance.

- Delivery success ratio: ratio of the number of geocast nodes which received the geocast packet to the number of all nodes in the geocast region

- Overhead: the average number of geocast packets received by each geocast node

- Lifetime of a geocast region: As a sensor node uses its energy to send/receive packets and sense data, its energy level decreases and it eventually loses all its energy to become a dead node. As time passes, the number of dead nodes increases and, therefore, the area covered by live nodes dwindles. We define the sensing area within a geocast region to be the union of the sensing range of all the active nodes within that geocast region. Then we define the lifetime of a geocast region 
to the time between the initial time of the geocast region and the time at which the ratio of the sensing area over the geocast region area falls below $80 \%$.

\subsection{Results and Analysis}

Figure 3 shows the delivery success rate of the algorithms. The flooding algorithm has the highest delivery success rate but we find that AC-MNF and EA-AC-MNF have almost similar success rate. Figure 4 shows the packet overhead of the algorithms. We see that the flooding algorithm has very high overhead while both AC-MNF and EA-AC-MNF has similarly low overhead. In the flooding algorithm all the nodes in the geocast region broadcast the geocast packet but in the other two algorithms only some carefully selected nodes can broadcast the geocast packet. Therefore, the flooding algorithm has higher delivery success rate but only at the cost higher packet overhead. The simulation results show that EA-AC-MNF has the lowest delivery success rate, but because usually sensor nodes cover mutually overlapping area, this small decrease in delivery success rate does affect the process of collecting data from the geocast region very much.

Figure 5 shows the lifetime of the algorithms. We see that the flooding algorithm has the shortest lifetime while the EA-AC-MNF algorithm has the longest lifetime. This is made possible because the proposed algorithm, EA-AC-MNF, considers the energy level of sensor nodes when selecting the sensor nodes which will forward geocast packets.

From the above performance results we conclude that the proposed algorithm, EA-AC-MNF has the longest lifetime while it has high delivery success rate and low overhead. This is made possible because the proposed algorithm tries to minimize the number of sensor nodes which forwards geocast commands and eliminates sensor nodes with low energy level when selecting command forwarders.

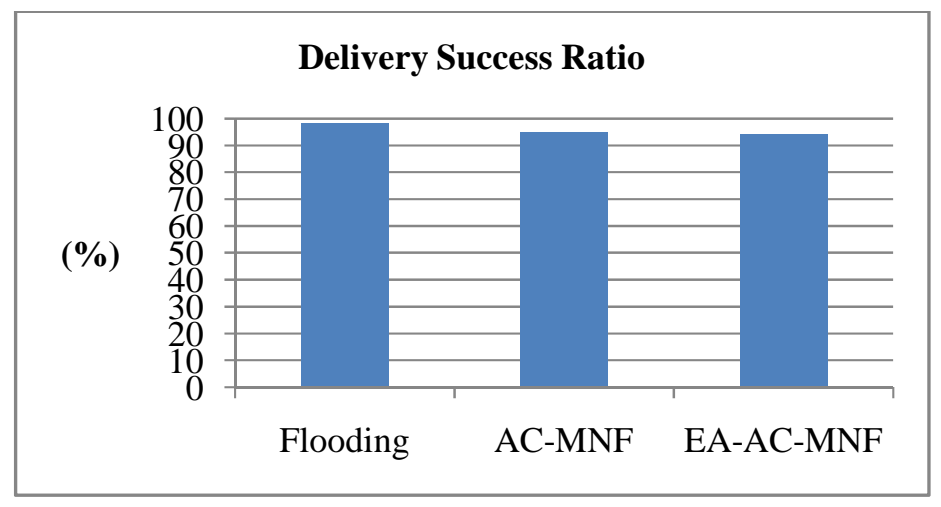

Figure 3. Delivery success ratio 


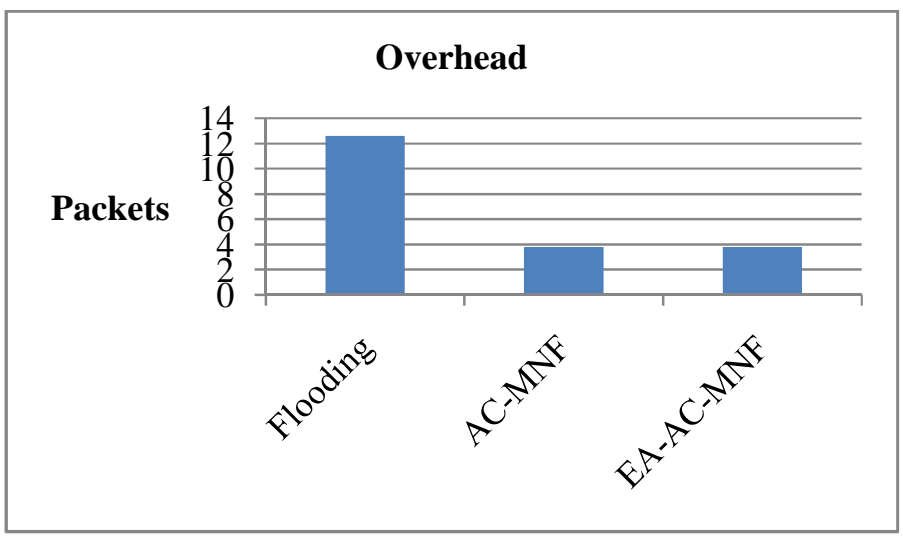

Figure 4. Overhead

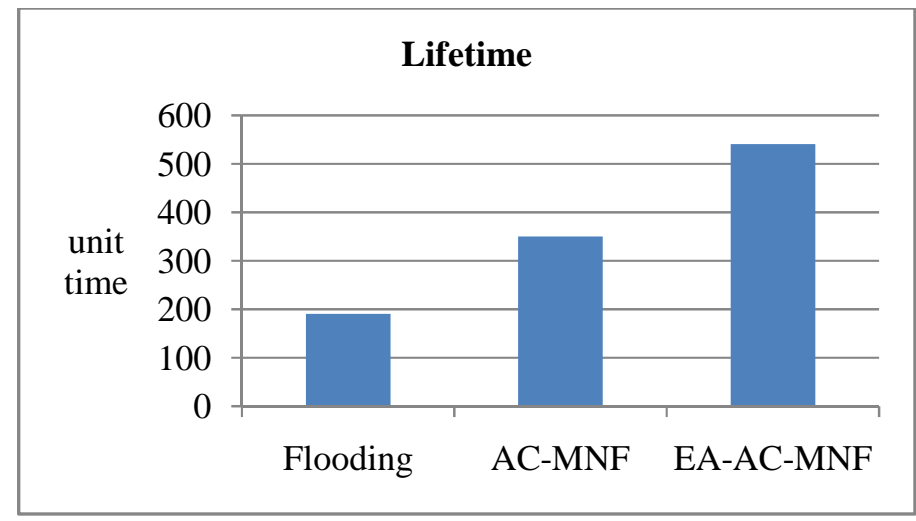

Figure 5. Lifetime of a geocast region network

\section{CONCLUSIONS}

Geocast is a mechanism which sends packets from a sink node to all the sensor nodes in a certain geographic area. Because sensor nodes are operating with limited power source, it is important to develop an energy efficient geocast protocol.

A Geocast protocol consists of two phases. In the first phase a geocast packet is unicast from the sink node to a node in the geocast region. In the second phase the packet is broadcast from that geocast node to all the nodes in the geocast region. In this paper we considered only the second phase and propose an energy efficient protocol to broadcast geocast packet with a geocast region. The proposed protocol tries to minimize the number of nodes forwarding the geocast packets with consideration into the energy level of nodes. The performance of the proposed protocol was compared with other algorithms through simulation. The results show that the proposed protocol is very energy efficient while having high delivery success rate and low overhead.

\section{ACKNOWLEDGEMENTS}

This work was supported by 2011 Hongik University Research Fund. 
International Journal of Computer Science \& Information Technology (IJCSIT) Vol 4, No 4, August 2012

\section{REFERENCES}

[1] J.Yick, B.Mukherjee \& D.Ghosal, "Wireless Sensor Network Survey", Computer Networks, vol.52, no.12, pp.2292-2330, 2008.

[2] P.Yao, E. Krohne \& T.Camp, "Performance Comparison of Geocast Routing Protocols for a MANET”, Proceedings of IEEE ICCCN, pp.213-220, 2004.

[3] Y.Yu, R.Govindan \& D.Estrin, "Geographical and Energy Aware Routing: A Recursive Data Dissemination Protocol for Wireless Sensor Networks", UCLA Technical Report, 2001.

[4] B.Williams \& T.Camp, "Comparison of Broadcasting Techniques for Mobile Ad Hoc Networks", Proceedings of MOBIHOC, pp.194-205, 2002.

[5] S.No, Y.Tseng, Y.Chen \& J.Sheu, "The Broadcast Strom Problem in a Mobile Ad Hoc Network", Proceedings of MOBICOM, pp.151-162, 1999.

[6] H.Lim et al, "Multicast Tree Construction and Flooding in Wireless Ad Hoc Networks", Proceedings of ACM MSWIN, 2000.

[7] W.Peng \& X.Lu, "On the Reduction of Broadcast Redundancy in Mobile Ad Hoc Networks", Proceedings of MOBIHOC, 2000.

[8] A.Qayyum, L.Viennot \& A.Laouiti, "Multipoint Relaying: An Efficient Technique of Flooding in Mobile Wireless Networks", INRIA Technical Report 3898, 2000.

[9] W.Peng \& X.Lu, "AHBP: An Efficient Broadcast Protocol for Mobile Ad Hoc Networks", Journal of Science and Technology, 2002.

[10] W.Peng \& X.Lu, "Efficient Broadcast in Mobile Ad Hoc Networks using Connected Dominating Sets", Journal of Software, 1999.

[11] J.Suced \& I.Marsic, "An Efficient Distributed Network-Wide Broadcast Algorithm for Mobile Ad Hoc Networks”, Rutgers University CAIP Technical Report 248, 2000.

[12] Y.C.Shim, “A New Geocast Protcol for a Moving Region in Sensor Networks", WSEAS Transactions on Communications, Vol 6, No. 4, 2007.

[13] Y.C.Shim \& H.Kang, "An Efficient Geocast Algorithm using 2-hop Neighbour Knowledge in Sensor Networks", International Journal on Latest Trends in Computing, Vol. 2, No. 4, 2011.

\section{Authors}

Young-Chul Shim received a Ph.D. in computer science from University of California, Berkeley. He is currently professor of the Department of Computer Engineering of Hongik University. His research interests are in the areas of wireless and mobile networks, Internet protocols, and cloud computing.

Sehun Noh received his MS degree in computer engineering from Hongik University. He currently works for GuardianNet in Seoul, Korea. 